\title{
Trapped phase-segregated Bose-Fermi mixtures and their collective excitations
}

\author{
Bert Van Schaeybroeck and Achilleas Lazarides \\ Instituut voor Theoretische Fysica, Katholieke Universiteit Leuven, Celestijnenlaan 200 D, B-3001 Leuven, Belgium
}

(Received 10 April 2008; revised manuscript received 17 September 2008; published 17 March 2009)

\begin{abstract}
Recent progress in the field of ultracold gases has allowed for the creation of phase-segregated Bose-Fermi systems. We present a theoretical study of their collective excitations at zero temperature. As the fraction of fermion-to-boson particle number increases, the collective mode frequencies take values between those for a fully bosonic and those for a fully fermionic cloud, with damping in the intermediate region. This damping is caused by fermions which are resonantly driven at the interface.
\end{abstract}

DOI: 10.1103/PhysRevA.79.033618

PACS number(s): 67.85.Pq, 03.75.Mn, 05.30.Jp

\section{INTRODUCTION}

Since their first experimental observation [1], degenerate boson-fermion $(\mathrm{BF})$ systems have been realized using more than five different particle mixtures [2]. The one composed of fermionic ${ }^{40} \mathrm{~K}$ and bosonic ${ }^{87} \mathrm{Rb}$ particles is the most thoroughly studied: Experiments have been performed on its collective excitations [3] and on collapse and expansion dynamics $[4,5]$. Using a Feshbach resonance to tune the interspecies interaction toward repulsion produced strong signs of phase segregation [6,7]; the presence of such resonances has also been observed in BF mixtures of different species [8]. Despite these advances, many new physical phenomena still await their experimental realization. In this context, it has been theoretically predicted that, for homogeneous systems, a multitude of phases appear on varying the interaction parameters [9]. In trapped systems, this gives rise to states with a large variety of spatial structures [10].

As is known from experiments with single-component Bose and Fermi systems, much physical information can be extracted by probing collective motions. Theoretically, the collective excitations of BF mixtures have been intensively studied for the phase-mixed BF system [11], including the regime close to phase demixing [12]. In Ref. [13], the collective excitations of phase-segregated Bose-Fermi mixtures across phase demixing were considered in the case of very small fermionic particle number.

In this work, we focus on the collective motions of isotropically trapped, fully segregated ${ }^{40} \mathrm{~K}-{ }^{87} \mathrm{Rb}$ mixtures. More specifically, we investigate the eigenfrequencies as a function of the fraction of the total particles that is fermionic, a parameter which is accessible in experiments. Using an exact analytic solution for the Boltzmann-Vlasov equation of a trapped, collisionless Fermi gas, we investigate monopole modes and their (collisionless) damping. To qualitatively investigate lower symmetry (multipole) modes we use a fully hydrodynamic description. This leads to the prediction of in-phase and out-of-phase modes, with similar oscillations of inner and outer boundaries. Whereas the breathing mode frequencies smoothly cross over from the purely fermionic to the purely Bose-Einstein condensate (BEC) spectrum, we find that the presence of a small fraction of fermions has a dominant effect on all other modes.

We consider in the following $N_{B}$ bosons and $N_{F}$ fermions, trapped by isotropic harmonic confinement $U_{i}(\mathbf{r})=m_{i} \omega_{i}^{2} r^{2} / 2$ with $i=B, F$; we shall take $m_{B} \omega_{B}^{2}=m_{F} \omega_{F}^{2}$ such that both particle species feel the same external potential, as is the case for the experiments on ${ }^{40} \mathrm{~K}$ and ${ }^{87} \mathrm{Rb}$ mixtures [3-6]. Within mean-field theory the equilibrium state of a BF mixture with chemical potentials $\mu_{B}$ and $\mu_{F}$ is fully characterized by the wave function $\psi_{B}$ of the Bose-Einstein condensate (BEC) and by the fermionic density $n_{F}$. The associated grand potential is $[9,10]$

$$
\begin{aligned}
\Omega= & \int d \mathbf{r} \psi_{B}^{*}\left(-\frac{\hbar^{2} \nabla^{2}}{2 m_{B}}+U_{B}-\mu_{B}\right) \psi_{B}+\frac{G_{B B}}{2}\left|\psi_{B}\right|^{4} \\
& +\left(U_{F}-\mu_{F}\right) n_{F}+3 G_{F F} n_{F}^{5 / 3} / 5+G_{B F} n_{F}\left|\psi_{B}\right|^{2}
\end{aligned}
$$

with $G_{F F}=\hbar^{2}\left(6 \pi^{2}\right)^{2 / 3} / 2 m_{F}, G_{B F}=2 \pi \hbar^{2} a_{B F}\left(m_{F}^{-1}+m_{B}^{-1}\right)$, and $G_{B B}=4 \pi \hbar^{2} a_{B B} / m_{B}$ the interspecies and bosonic intraspecies coupling constant, respectively. Minimization of this grand potential with respect to $n_{F}(\mathbf{r})$ and $\psi_{B}(\mathbf{r})$ gives the ThomasFermi (TF) and Gross-Pitaevskii (GP) equations. When, however, the particle number is sufficiently large, the ground-state configuration is well described using a localdensity approximation. Therefore, each point $\mathbf{r}$ in the trap can be treated locally, using the effective chemical potentials $\mu_{i}(\mathbf{r})=\mu_{i}-U_{i}(\mathbf{r})$, where $\mu_{i} \equiv m \omega_{i}^{2} R_{i}^{2} / 2$ is the chemical potential at the center of the trap and $i=B, F$.

Full BF phase segregation appears for sufficiently large interspecies repulsion; more specifically, it occurs when $G_{B F}$ is larger than $G_{B B} \mu_{F} / \mu_{B}$ at the BF overlap region. For all the ${ }^{40} \mathrm{~K}-{ }^{87} \mathrm{Rb}$ trap configurations considered here, this requires the interspecies scattering length $a_{B F}$ to be larger than $10^{3}$ Bohr, which is well below the experimentally reported values of Ref. [7]. The phase-segregated BF trap then consists of a purely bosonic core surrounded by a purely fermionic shell; in between there is a spherical shell where the bosons and fermions overlap. The thickness of this shell is generally very thin, of the order of the BEC healing length $\hbar /\left(2 m_{B} \mu_{B}\right)^{1 / 2}$. For almost all our further considered configurations this length is much smaller than the trapping length scales $\hbar / m_{i} \omega_{i}$ with $i=B, F$.

The influence of a thin interface on the equilibrium configuration can be incorporated through an interface tension $\sigma$, an exact expression for which is given in Eq. (A2) in the Appendix. Mechanical equilibrium at the interface implies that the pressures $P_{i}$ on either side of the interface are related by Laplace's formula, 

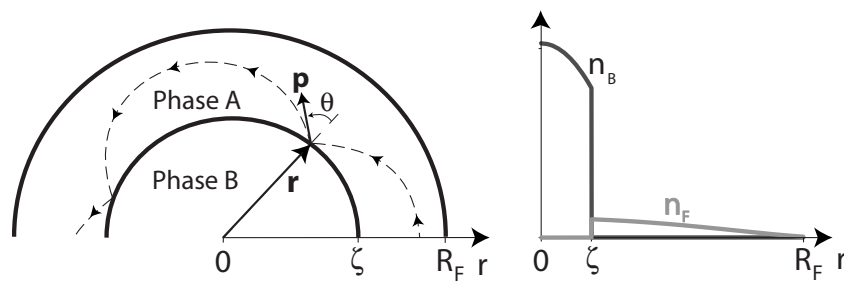

FIG. 1. (Left) Cross section of trap. Fermions and bosons are separated by the interface at radial position $\zeta$. Fermions follow trajectories along the dashed line and reflect off the interface. (Right) Boson and fermion densities $n_{B}$ and $n_{F}$ against radial position.

$$
P_{B}-P_{F}=2 \sigma / \zeta .
$$

Here, $\zeta$ is the radial position of the interface (see Fig. 1). Finally the particle numbers of the BEC core and fermion shell are

$$
\begin{gathered}
N_{B}=\frac{R_{B}^{5}\left(m_{B} \omega_{B} / \hbar\right)^{2}}{2 a_{B}}\left(\frac{\zeta^{3}}{3 R_{B}^{3}}-\frac{\zeta^{5}}{5 R_{B}^{5}}\right), \\
N_{F}=\frac{\left(m_{F} \omega_{F} R_{F}^{2} / \hbar\right)^{3}}{72 \pi}\left[\mathcal{K}(1)-\mathcal{K}\left(\zeta / R_{F}\right)\right],
\end{gathered}
$$

where $\mathcal{K}(x) \equiv x \sqrt{1-x^{2}}\left(14 x^{2}-3-8 x^{4}\right)+3 \arcsin (x)$.

The GP and TF equations, together with the number equations (2) and the condition for mechanical equilibrium at the interface (1), allow us then to fully determine the equilibrium trap configuration.

Having established the equilibrium configuration of the BF system, we now proceed to the description of its dynamics. We start by discussing the dynamics of a BEC, which, as is well known, is governed by the equations of hydrodynamics. Then, both collisionless and hydrodynamical behavior is considered for the outer Fermi shell. While a collisionless treatment is experimentally more relevant, the succeeding hydrodynamic consideration allows us to bring out some important qualitative aspects of the system's behavior. By combining the fermionic and bosonic dynamics by means of the appropriate boundary conditions, we then extract collective mode frequencies.

\section{DYNAMICS OF THE BEC}

As is usual, collective excitations are treated in a hydrodynamical description by considering small-amplitude density oscillations $\delta n_{B}$ which linearize the continuity, $\partial_{t} \delta n_{B}$ $+\boldsymbol{\nabla} \cdot\left(n_{B} \mathbf{v}_{B}\right)=0$, and Euler, $m_{B} n_{B} \partial_{t} \mathbf{v}_{B}=\boldsymbol{\nabla} \delta P_{B}-n_{B} \boldsymbol{\nabla} U_{B}$, equations. Here, $n_{B}=\left|\psi_{B}\right|^{2}$ is the BEC density. Using standard methods $[14,15]$, the chemical potential oscillation $\delta \mu_{B}$ with temporal frequency $\omega$ then satisfies

$$
m_{B} n_{B} \omega^{2} \delta \mu_{B}+\mu_{B} \nabla \cdot\left(n_{B} \nabla \delta \mu_{B}\right)=0,
$$

the solution to which is $\delta \mu_{B} \propto Y_{\ell}^{m} r^{\ell} F\left(\alpha^{+}, \alpha^{-}, \ell\right.$ $\left.+3 / 2,\left(r / R_{B}\right)^{2}\right)$, with $F$ the hypergeometric function, $Y_{\ell}^{m}$ the spherical harmonic, $2 \alpha^{ \pm}=z \pm\left[z^{2}+2\left(\omega^{2} / \omega_{B}^{2}-\ell\right)\right]^{1 / 2}, \quad z=\ell$ $+1 / 2+1$, and $\ell$ a positive integer or zero.

Here, we must impose a boundary condition at the BF interface. Since we are assuming that the oscillations occur at local thermodynamic equilibrium [16], we conclude that the two fluids are completely immiscible and the interface impermeable. This immediately leads to the condition

$$
\dot{\zeta}=\left.\boldsymbol{e}_{\zeta} \cdot \mathbf{v}_{B}\right|_{\zeta},
$$

in which $\boldsymbol{e}_{\zeta}$ is a unit radial vector normal to the interface, and the dot denotes the derivative with respect to time. That is, the interface velocity must be equal to the $\mathrm{BEC}$ velocity at the interface, ensuring vanishing of the flux of bosons through the interface.

\section{COLLISIONLESS FERMI GAS}

If the fermionic particles are collisionless, as expected at low temperatures, the appropriate dynamical description is the Boltzmann-Vlasov equation with a vanishing collision integral,

$$
\partial_{t} f+\boldsymbol{v} \cdot \nabla_{\mathbf{r}} f-\omega_{F}^{2} \mathbf{r} \cdot \nabla_{\mathbf{v}} f=0 .
$$

The mean-field interaction term is also absent, again because fermionic interactions are suppressed by Pauli blocking. Since collisions do not act to restore local equilibrium during the oscillation, the Fermi sphere is deformed during the oscillation. We therefore write $f=f_{0}+\delta\left(|\boldsymbol{p}|-p_{f}\right) \nu(r, \chi) e^{-i \omega t}$, where $p_{f}(\mathbf{r})=\left[2 m_{F} \mu_{F}(\mathbf{r})\right]^{1 / 2}$ is the local Fermi momentum and $\chi=\cos \theta$ with $\theta$ the angle between $\mathbf{r}$ and $\mathbf{p}$ (see Fig. 1). This parametrization is the most convenient for studying spherically symmetric oscillations. Equation (5) then becomes

$$
-i \omega \nu+\omega_{F} \tilde{p} \chi \partial_{r} \nu+\omega_{F}\left(1-\chi^{2}\right) g(r) \partial_{\chi} \nu=0,
$$

with $g(r)=\tilde{p} / r-r / \tilde{p}$ and $\tilde{p}^{2} \equiv R_{F}^{2}-r^{2}$. The general solution to Eq. (6) can readily be found,

$$
\nu(r, \chi)=\mathcal{C}\left[r^{2}\left(R_{F}^{2}-r^{2}\right)\left(1-\chi^{2}\right)\right] e^{i \omega \tau / 2},
$$

with $\mathcal{C}[x]$ an arbitrary function of the variable $x$ [17], and

$$
\tau(r, \chi)=\arctan [2 \chi / g(r)] / \omega_{F} .
$$

Physically, $\tau(\zeta, \chi)$ is the time for a particle, leaving the interface at an angle $\theta=\arccos (\chi)$ and speed $p_{f}(\zeta) / m_{F}$, to return to it.

To fix the arbitrary function $\mathcal{C}$ in the general solution of Eq. (7), we must once more impose the appropriate boundary condition. As already explained, the interface is completely impermeable to both particle types; incident fermions are therefore specularly reflected [18]. A boundary condition encoding this process may be derived using the following arguments, after Bekarevich and Khalatnikov [19]: First, in a frame moving with the interface, the energy of the incident and reflected excitations must be equal; second, the influx of particles in this frame must be equal to their outflux. These two conditions result in [15]

$$
\nu(\zeta, \chi)-\nu(\zeta,-\chi)=2 m_{F} \chi \dot{\zeta}
$$

where $\dot{\zeta}$ is the velocity of the interface. One may understand Eq. (9) as follows: For a stationary interface, $\dot{\zeta}=0$, all particles incident at angle $\theta$ will be reflected such that $\nu(\chi)$ 
$=\nu(-\chi)$. If, on the other hand, the interface is moving with $\dot{\zeta}>0$, then $\nu(\chi)-\nu(-\chi)$ is positive and proportional to $\chi$; that is, (a) the reflected state at any given angle becomes more highly occupied than the incident state at the same angle, and (b) the difference in occupancy is largest when $\chi=1$, that is, for particles incident normal to the interface. Thus, there is an overall probability transfer to states closer to normal $(\chi=1)$. This is due to the momentum transfer from the interface to the reflected particles. At $r=\zeta$, the solution of the Boltzmann-Vlasov equation (7) which satisfies condition (9) is

$$
\nu(\zeta, \chi)=m_{F} \chi \dot{\zeta}[1-i \cot (\omega \tau / 2)] .
$$

Demanding local mechanical equilibrium at the interface results in the linearized Laplace equation with the role of the fermionic pressure fluctuation played by the radial component of the momentum flux tensor $\delta \Pi_{r r}^{F}=\int d^{3} \mathbf{p}(\chi p)^{2}$ $\left(f-f_{0}\right) /\left[m_{F}(2 \pi \hbar)^{3}\right]$. Thus,

$$
\begin{aligned}
n_{B} \delta \mu_{B}-\delta \Pi_{r r}^{F}= & \sigma \delta \zeta(\ell-1)(\ell+2) / \zeta^{2}-3 \sigma \delta \zeta m \omega_{F}^{2} / \mu_{B} \\
& +3 \sigma \delta \mu_{B} /\left(\mu_{B} \zeta\right)-\delta \zeta \partial_{r}\left(P_{B}-P_{F}\right),
\end{aligned}
$$

with all quantities evaluated at the interface and $\delta \zeta$ $\propto Y_{\ell}^{m}(\theta, \phi)$ the departure of the interface from its equilibrium position. Solving Eqs. (11) and (4) for $\omega$ fixes the eigenfrequencies.

\section{DAMPING}

Despite the absence of collisions in the fermionic gas, damping of the collective oscillation may occur, induced by the interaction of the fermions with the interface. To understand its origin, consider the two time scales relevant in the collisionless fermionic phase: The period of oscillation $2 \pi / \omega$, and $\tau$, the time of flight for a particle projected at angle $\theta=\arccos (\chi)$ from the interface [see Eq. (8)]. From Eq. (10), $\nu(\zeta, \chi)$ diverges if

$$
\tau(\zeta, \chi)=2 \pi n / \omega,
$$

with $n$ an integer; that is, when there exist particles which depart from and return to the interface in exactly an integer number of oscillation periods. The resulting pole on the integration path in the pressure tensor $\delta \Pi_{r r}^{F}$ and thus also in the dispersion relation, Eq. (11), must be avoided by some definite prescription [20]. The appropriate manner is by deforming the contour of integration for $\delta \Pi_{r r}^{F}$ so as to pass below the pole [21]. This gives rise to an additional, imaginary term due to the residue in $\delta \Pi_{r r}^{F}$; in this way, the solution for $\omega$ to the dispersion relation acquires a negative imaginary part: Damping occurs.

Can one intuitively understand when damping occurs? In other words, under which conditions are there particles which satisfy Eq. (12)? As we will see, whether damping occurs or not depends solely on the interface position and the real part of $\omega, \operatorname{Re}(\omega)$, and this remains true for the multipole modes.

For fixed interface position $\zeta / R_{F}$, the time $\tau$ takes values only within a restricted interval [Fig. 2(a)]. Consider first

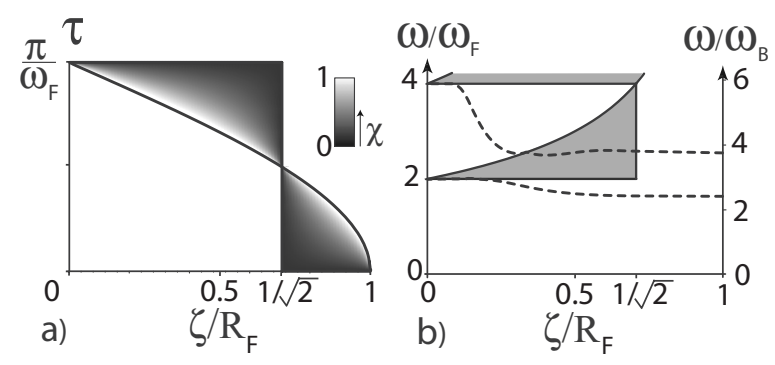

FIG. 2. (a) The full lines outline the region of possible values of $\tau$ [see Eq. (8)] as a function of the interface position $\zeta$. (b) For any multipole mode, damping will occur for values of $\operatorname{Re}(\omega)$ and $\zeta$ in the shaded region. The dashed lines correspond to the monopole modes depicted in Fig. 3 with full lines.

radially moving particles $(\chi=1)$; for vanishing $\zeta / R_{F}$, they take one-half a trapping period to return to the interface, i.e., $\tau(0,1)=\pi / \omega_{F}$ and, as $\zeta$ is increased, $\tau$ smoothly vanishes as the interface approaches the trap edge. For $\zeta / R_{F}>1 / \sqrt{2}$, particles projected parallel to the interface $(\chi=0)$ experience a "centrifugal force" which is insufficient to overcome the harmonic trapping force such that $\tau(\zeta, 0)=0$; for general $\chi$, one finds $0<\tau(\zeta, \chi)<\tau(\zeta, 1)$. Conversely, if $\zeta / R_{F}<1 / \sqrt{2}$, a particle with $\chi=0$ performs exactly one-half an orbit between two collisions with the interface and therefore $\tau(\zeta, 0)$ $=\pi / \omega_{F}$; in this case, $\tau(\zeta, 1)<\tau(\zeta, \chi)<\pi / \omega_{F}$ for general $\chi$.

From the possible values of $\tau$, it is straightforward to obtain the values of $\omega$ and $\zeta$ for which damping occurs [see shaded region in Fig. 2(b)]. For small $\zeta / R_{F}$, we have seen that $\tau \approx \pi / \omega_{F}$; but this is exactly one-half the period of a collisionless Fermi gas. Damping is therefore expected for the lowest monopole mode when $N_{B} \ll N_{F}$. On the other hand, when $\zeta / R_{F} \approx 1$, due to a vanishing $\tau$, Eq. (12) is satisfied only for large values of $\omega$. This inhibits damping for the lowest modes in the case $N_{F} \ll N_{B}$.

The damping we observe is somewhat analogous to Landau damping, which corresponds to energy transfer from collective modes into single-particle incoherent modes. In our case, no clear distinction exists between collective and single-particle modes in the Fermi gas and energy goes into incoherent excitations in the collisionless gas.

\section{RESULTS}

We consider trap configurations containing $10^{6}$ particles and characterized by the parameters $a_{B B}=5.23 \mathrm{~nm}$ and $\omega_{B}$ $=570 \mathrm{~Hz}$ [22]. In Fig. 3, we show with full lines the real part of the frequencies of the two lowest monopole modes as a function of the Fermi fraction $Q=N_{F} /\left(N_{B}+N_{F}\right)$. The gray and black lines indicate the damped and undamped frequencies, respectively. The lowest monopole mode smoothly crosses over between the value $\sqrt{5} \omega_{B}$ for a fully superfluid bosonic trap at $Q=0$, to the value $2 \omega_{F}$ of the fully collisionless (fermionic) trap at $Q=1$ [14]. As explained earlier, the lowest monopole mode is damped when $\zeta / R_{F} \approx 0$; indeed, for $Q>0.85$, very weak damping is present with a maximum damping rate of about $10^{-3} \omega_{B}$. As expected, the second monopole mode frequency goes over from $\sqrt{14} \omega_{B}$ at $Q=0$ to 


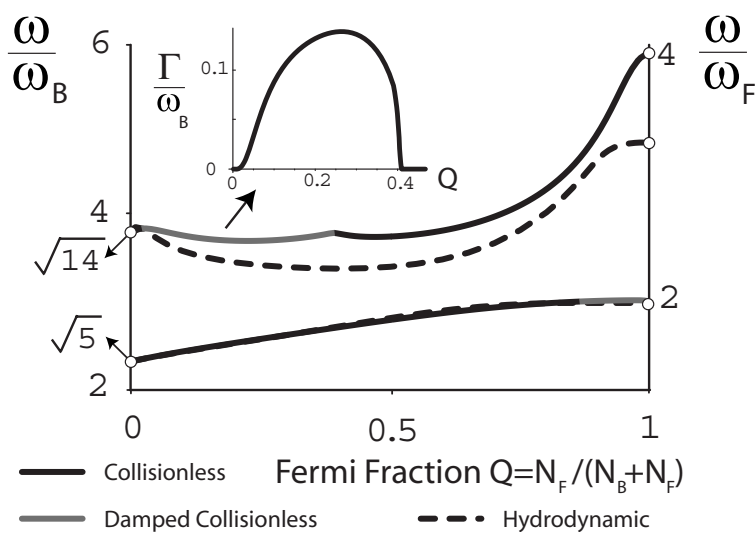

FIG. 3. The frequencies $\omega$ of the $\ell=0$ monopole modes as a function of the Fermi fraction $Q=N_{F} /\left(N_{B}+N_{F}\right)$. The full black and gray lines correspond to damped and undamped monopole modes, respectively, both calculated using collisionless fermion dynamics. The inset shows the damping rate $\Gamma$ of the second monopole mode. Dashed lines are calculated using a hydrodynamic Fermi shell.

$4 \omega_{F}$ at $Q=1$ [14]. Its variation, however, is nonmonotonic and considerable damping occurs in the interval $0.02<Q$ $<0.4$; the associated damping rate $\Gamma$ is shown in the inset of Fig. 3. The absence of damping for low values of $Q$ is in full agreement with the explanation given above. In Fig. 2(b), we plot the evolution of $\omega$ as a function of $\zeta$ (dashed lines) for both monopole modes; damping occurs in the shaded region.

\section{OUT-OF-PHASE MODES}

While a collisionless treatment is experimentally more relevant, the following hydrodynamic considerations for the fermions allow us to bring out some important qualitative aspects of the system's dynamical behavior.

The bulk solutions to the continuity and Euler equations for fermions are similar to those for the BEC [15]. The Laplace Eq. (11) used for the collisionless case still holds but with $\delta \Pi_{r r}^{F}$ replaced by $n_{F} \delta \mu_{F}$ and the impermeability of the interface implies $\dot{\delta} \zeta=\left.\mathbf{e}_{\zeta} \cdot \mathbf{v}_{F}\right|_{\zeta}$.

In Fig. 3, we show with dashed lines the frequencies for the breathing modes $\ell=0$ using a hydrodynamical Fermi shell. In Fig. 4, on the other hand, the lowest multipole $(\ell \neq 0)$ modes are shown. Both the multipole modes in which the interface and outer boundary move in phase (full lines) and out of phase (dashed lines) are present [15]. Both the in-phase (IP) and out-of-phase (OOP) modes have in-phase velocities at the $\mathrm{BF}$ interface and are both generic to a twocomponent system $[15,23]$. An interesting result is that the frequencies of all the $\ell \neq 0$ modes vary strongly at small $Q$ and remain approximately constant at larger $Q$; this is in contrast with the monopole modes.

\section{DISCUSSION}

The mass difference between the ${ }^{40} \mathrm{~K}$ and ${ }^{87} \mathrm{Rb}$ particles may cause a gravitational sag to be present. Incorporating such an effect is beyond the scope of this work. However, we expect gravity not to affect the validity of our qualitative

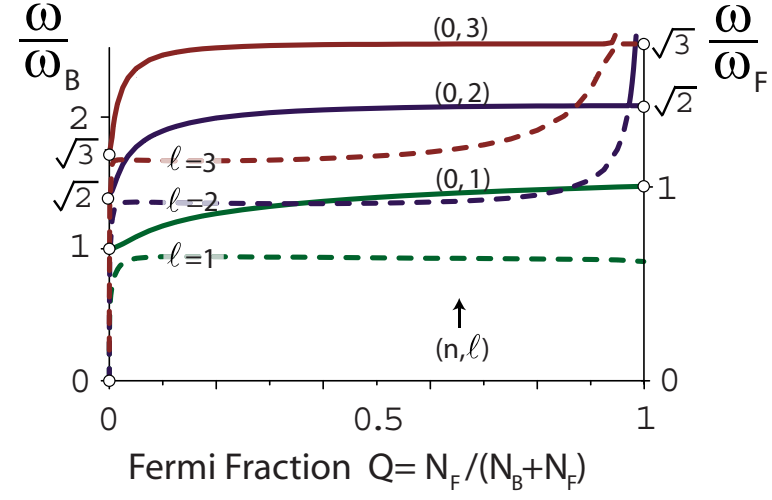

FIG. 4. (Color online) The frequencies $\omega$ of the multipole $(\ell \neq 0)$ modes as a function of the Fermi fraction $Q=N_{F} /\left(N_{B}\right.$ $\left.+N_{F}\right)$. All are found using a fully hydrodynamical trap and involve in-phase (full lines) and out-of-phase (dashed lines) motions of inner and outer boundaries.

results such as the damping mechanism and the presence of out-of-phase modes. Note also that the gravitational sag may be quenched experimentally by counterbalancing external potentials or by tight radial confinement.

A finite temperature will change the collective mode frequencies mostly through a change in the fermion dynamics. We expect $\operatorname{Re}(\omega)$ to be changed little with temperature, while damping is expected to occur for a broader range of $Q$ and occurs by the same mechanism as described here [24].

Our approach fails when the interface is as thin as the outer fermion shell; we find that this happens for Fermi fractions below $2 \times 10^{-3}$. In that case, damping of the collective excitations happens by usual Landau damping throughout the BF overlap region [12]. Our work is complementary to Ref. [12] in the sense that they study the process of phase demixing for a Fermi fraction $4 \times 10^{-5}$ whereas we focus on the variation of the Fermi fraction in the case of complete phase segregation.

\section{CONCLUSION}

We present monopole mode frequencies for the trapped phase-segregated ${ }^{40} \mathrm{~K}-{ }^{87} \mathrm{Rb}$ mixture. A damping mechanism is identified which is generic to all collective modes. Within a fully hydrodynamic approximation we then obtain mode frequencies for modes involving out-of-phase and in-phase motions of inner and outer boundaries. All of our results are only very weakly dependent on the surface tension.

\section{ACKNOWLEDGMENTS}

We acknowledge partial support by Contract No. FWO G.0115.06; A.L. is supported by Contract No. GOA/2004/02 and B.V.S. by the Research Fund K.U. Leuven.

\section{APPENDIX: INTERFACE TENSION}

In order to obtain the surface tension of a BF interface, one may consider an infinite space containing fermions at $x$ $\rightarrow-\infty$ and bosons at $x \rightarrow+\infty$, bound by a flat interface which 
is parallel to the $y-z$ plane. Both species are taken to be untrapped $\left(U_{B}=U_{F}=0\right)$ and at fixed chemical potentials $\mu_{B}$ and $\mu_{F}$. The appropriate boundary conditions for $\psi_{B}(x)$ and $n_{F}(x)$ are $\psi_{B}(-\infty)=n_{F}(+\infty)=0$ while $\psi_{B}(+\infty)=\sqrt{\mu_{B} / G_{B B}}$ and $n_{F}(-\infty)=\left(\mu_{F} / G_{F F}\right)^{3 / 2}$. We define the BEC coherence length $\xi_{B} \equiv \hbar /\left(2 m_{B} \mu_{B}\right)^{1 / 2}$ and the dimensionless parameters $\kappa^{2}$ $\equiv G_{B B} \mu_{F} / G_{B F} \mu_{B}$ and $\phi_{B}(x) \equiv \psi_{B}(x) / \sqrt{\mu_{B} / G_{B B}}$. Using these variables, it is useful to introduce the first integral associated with the GP and TF equations for our system under consideration,

$$
2 \xi_{B}^{2}\left(\partial \phi_{B} / \partial x\right)^{2}=\left(1-\phi_{B}^{2}\right)^{2}-\mathcal{G}\left(\kappa, \phi_{B}\right),
$$

where $\mathcal{G}\left(\kappa, \phi_{B}\right)=\left(1-\phi_{B}^{2} / \kappa^{2}\right)^{5 / 2} \Theta\left(\kappa-\phi_{B}\right)$ and $\Theta$ the Heaviside function. Using Eq. (A1), the interface tension which is the excess grand potential per unit area of the interface can be recast in the form

$$
\sigma=4 P \xi_{B}^{2} \int_{-\infty}^{\infty} d x\left(\partial \phi_{B} / \partial x\right)^{2}
$$

Using the GP and TF equations and the transformation $d x$ $=\left(\partial \phi_{B} / \partial x\right)^{-1} d \phi_{B}$, the interface tension $\sigma$ associated with a flat BF interface is

$$
\sigma(\kappa)=2 \sqrt{2} P \xi_{B} \int_{0}^{1} d \phi_{B} \sqrt{\left(1-\phi_{B}^{2}\right)^{2}-\mathcal{G}\left(\kappa, \phi_{B}\right)} .
$$

The surface tension is minimal at $\kappa=1 \quad\left(\sigma=0.52 P \xi_{B}\right)$ and reaches its maximal value $4 \sqrt{2} P \xi_{B} / 3$ when $\kappa=0$. Note that one may use this exact result for the surface tension in a trap when the chemical potentials $\mu_{B}$ and $\mu_{F}$ are evaluated at the interface.
[1] A. G. Truscott et al., Science 291, 2570 (2001); F. Schreck, L. Khaykovich, K. L. Corwin, G. Ferrari, T. Bourdel, J. Cubizolles, and C. Salomon, Phys. Rev. Lett. 87, 080403 (2001).

[2] Z. Hadzibabic, C. A. Stan, K. Dieckmann, S. Gupta, M. W. Zwierlein, A. Gorlitz, and W. Ketterle, Phys. Rev. Lett. 88, 160401 (2002); C. Silber, S. Gunther, C. Marzok, B. Deh, P. W. Courteille, and C. Zimmermann, ibid. 95, 170408 (2005); J. M. McNamara, T. Jeltes, A. S. Tychkov, W. Hogervorst, and W. Vassen, ibid. 97, 080404 (2006).

[3] G. Modugno, M. Modugno, F. Riboli, G. Roati, and M. Inguscio, Phys. Rev. Lett. 89, 190404 (2002); F. Ferlaino, R. J. Brecha, P. Hannaford, F. Riboli, G. Roati, G. Modugno, and M. Inguscio, J. Opt. B: Quantum Semiclassical Opt. 5, S3 (2003).

[4] C. Ospelkaus, S. Ospelkaus, K. Sengstock, and K. Bongs, Phys. Rev. Lett. 96, 020401 (2006); G. Modugno, G. Roati, F. Riboli, F. Ferlaino, R. J. Brecha, and M. Inguscio, Science 297, 2240 (2002).

[5] F. Ferlaino, E. deMirandes, G. Roati, G. Modugno, and M. Inguscio, Phys. Rev. Lett. 92, 140405 (2004); G. Roati, F. Riboli, G. Modugno, and M. Inguscio, ibid. 89, 150403 (2002).

[6] M. Zaccanti, C. D’Errico, F. Ferlaino, G. Roati, M. Inguscio, and G. Modugno, Phys. Rev. A 74, 041605(R) (2006).

[7] S. Ospelkaus, C. Ospelkaus, L. Humbert, K. Sengstock, and K. Bongs, Phys. Rev. Lett. 97, 120403 (2006).

[8] B. Deh, C. Marzok, C. Zimmermann, and P. W. Courteille, Phys. Rev. A 77, 010701(R) (2008); C. A. Stan, M. W. Zwierlein, C. H. Schunck, S. M. F. Raupach, and W. Ketterle, Phys. Rev. Lett. 93, 143001 (2004); S. Inouye, J. Goldwin, M. L. Olsen, C. Ticknor, J. L. Bohn, and D. S. Jin, ibid. 93, 183201 (2004).

[9] S. Powell, S. Sachdev, and H. P. Büchler, Phys. Rev. B 72, 024534 (2005); F. M. Marchetti, C. Mathy, D. A. Huse, and M. M. Parish, ibid. 78, 134517 (2008); L. Salasnich and F. Toigo, Phys. Rev. A 75, 013623 (2007); L. Viverit, C. J. Pethick, and H. Smith, ibid. 61, 053605 (2000); L. Viverit and S. Giorgini, ibid. 66, 063604 (2002).

[10] K. Mølmer, Phys. Rev. Lett. 80, 1804 (1998); R. Roth, Phys.
Rev. A 66, 013614 (2002); N. Nygaard and K. Mølmer, ibid. 59, 2974 (1999); Z. Akdeniz, A. Minguzzi, P. Vignolo, and M. P. Tosi, ibid. 66, 013620 (2002); X. X. Yi and C. P. Sun, ibid. 64, 043608 (2001); A. Minguzzi and M. P. Tosi, Phys. Lett. A 268, 142 (2000); S. Röthel and A. Pelster, Eur. Phys. J. B 59, 343 (2007).

[11] T. Maruyama and G. F. Bertsch, Phys. Rev. A 77, 063611 (2008); T. Maruyama, H. Yabu, and T. Suzuki, ibid. 72, 013609 (2005); T. Miyakawa, T. Suzuki, and H. Yabu, ibid. 62, 063613 (2000); M. Rodríguez, P. Pedri, P. Törmä, and L. Santos, ibid. 69, 023617 (2004); X.-J. Liu and H. Hu, ibid. 67, 023613 (2003); D. H. Santamore, S. Gaudio, and E. Timmermans, Phys. Rev. Lett. 93, 250402 (2004); M. Wouters, J. Tempère, and J. T. Devreese, Phys. Rev. A 66, 043414 (2002).

[12] P. Capuzzi, A. Minguzzi, and M. P. Tosi, Phys. Rev. A 68, 033605 (2003); 67, 053605 (2003); P. Capuzzi and E. S. Hernández, ibid. 64, 043607 (2001); T. Sogo, T. Miyakawa, T. Suzuki, and H. Yabu, ibid. 66, 013618 (2002).

[13] T. Sogo, T. Suzuki, and H. Yabu, Phys. Rev. A 68, 063607 (2003).

[14] C. J. Pethick and H. Smith, Bose-Einstein Condensation in Dilute Gases (Cambridge University Press, Cambridge, 2002); L. P. Pitaevskii and S. Stringari, Bose-Einstein Condensation (Clarendon, Oxford, 2003).

[15] A. Lazarides and B. Van Schaeybroeck, Phys. Rev. A 77, 041602(R) (2008).

[16] This is why we can use the equation of state, for example.

[17] Note that the paths along which the squared angular momentum $r^{2}\left(R_{F}^{2}-r^{2}\right)\left(1-\chi^{2}\right) m_{F}^{2} \omega_{F}^{2}$ is constant, corresponds to classical orbits in a harmonic potential.

[18] B. Van Schaeybroeck and A. Lazarides, Phys. Rev. Lett. 98, 170402 (2007).

[19] I. L. Bekarevich and I. M. Khalatnikov, Zh. Eksp. Teor. Fiz. 39, 1699 (1960) [Sov. Phys. JETP 12, 1187 (1961)].

[20] L. Landau, J. Phys. (USSR) 10, 25 (1946); N. A. Krall and A. W. Trivelpiece, Principles of Plasma Physics (McGraw-Hill, New York, 1973); P. M. Bellan, Fundamentals of Plasma Physics (Cambridge University Press, Cambridge, 2006).

[21] The validity of this method can be justified by repeating the 
calculations using Laplace, rather than Fourier, transforms in time.

[22] This frequency is the geometrical mean of the experimental frequencies of Ref. [4].
[23] A. A. Svidzinsky and S. T. Chui, Phys. Rev. A 67, 053608 (2003); 68, 013612 (2003).

[24] B. Van Schaeybroeck and A. Lazarides, e-print arXiv:0807.3636. 